\title{
Reduced kinetic description of electron multiplication in gases
}

\author{
L Friedland and Yu M Kagan \\ Centre for Plasma Physics, Racah Institute of Physics, The Hebrew University of Jerusalem. \\ Jerusalem, Israel
}

Received 19 March 1985, in final form 21 October 1985

\begin{abstract}
A one-dimensional model of electron multiplication in a gas is considered by using a system of moments equations. An approximate expression for the first Townsend ionisation coefficient is derived and found to be in good agreement with the experimental data for a variety of atomic gases.
\end{abstract}

\section{Introduction}

Consider a stationary, spatial electron current growth in a gas in a non-uniform electric field $\boldsymbol{E}=E(x) \hat{e}_{x}$. Assume that current density $j_{0}$ is initiated at plane $x=0$. As a result of the acceleration of the electrons in the electric field and a subsequent avalanche ionisation, the current increases with $x$ and can be represented by $j(x)=P(x) j_{0}$. The problem of the evaluation of $P(x)$ in strong uniform fields was studied on the basis of a onedimensional model (Friedland 1974, Friedland and Kagan 1982, 1983) in which the electrons were allowed to move strictly in the direction of the field. The energy losses in elastic collisions were neglected and it was assumed that new electrons had been created in ionising collisions, having identical initial energy. Also, therefore, because of the uniformity of the electric field, it was concluded that any average characteristic of a test electron, created in a gas at $x^{\prime}$, was a function of the distance $x-x^{\prime}$ only. For example, the average energy $\bar{w}$ of the test electron and the average number of ionisations $\bar{\alpha} \mathrm{d} x$ caused by the electron in the interval $(x, x+\mathrm{d} x)$ are both functions of $x-x^{\prime}$ only. Since the growing current in the discharge comprises an ensemble of test electrons, each with a similar average history, one can relate $P(x)$ to $\bar{\alpha}$. It was found indeed (Friedland 1974) that

$$
\frac{\mathrm{d} P}{\mathrm{~d} x}=-\int_{0}^{x} P\left(x^{\prime}\right) \frac{\partial \bar{\alpha}\left(x-x^{\prime}\right)}{\partial x} \mathrm{~d} x^{\prime} \quad P(0)=1 .
$$

Thus the total electron current can be expressed by a single test electron average characteristic $\bar{\alpha}\left(x-x^{\prime}\right)$ which can be conveniently found by using, for example, the Monte Carlo simulation method. Alternatively (Friedland and Kagan 1983) the Laplace transformation of (1) yields

$$
P_{s}=1 / s\left(1-\bar{\alpha}_{s}\right)
$$

where $P_{s}$ and $\alpha_{s}$ are Laplace images of $P(x)$ and $\bar{\alpha}(x)$ respectively. Therefore, if $\bar{\alpha}_{s}$ is known, the inverse Laplace transformation of (2) yields $P(x)$. Unfortunately, it is 
impossible to derive an analytic form of $\dot{\alpha}_{s}$ for an arbitrary dependence of the inelastic collision cross sections on the electron energy $\varepsilon$. Nevertheless, by assuming that the ionisation cross section rises linearly for small values of $\varepsilon$ and becomes a constant at $\varepsilon \rightarrow \infty$, expressions for $\alpha_{s}$ are reduced for the limiting cases of low and high values of the strength of the electric field $E$. The intermediate range of $E$ has been described by using simple interpolation formulae for $\alpha_{s}$ in the Laplace space. This procedure yields a good agreement with the experimental results for the current growth in a variety of different gases. The form of the interpolation, however, it not unique which is a disadvantage of the method.

In the present study we will consider the intermediate range of $E$ without employing the above-mentioned interpolation procedure. We will adopt the one-dimensional scattering model and use the Boltzmann equation for the conventional velocity distribution function $f(v, x)$ of the electrons in the avalanche $(\$ 2)$. Instead of solving for $f(v, x)$, we will derive and solve a system of equations for the moments of $f$. The exact solution is possible $(\$ 3)$ if one assumes a linear dependence of the inelastic cross sections on the electron energy $\varepsilon$. The case of more intense electric fields will be considered in $\S 4$ by including small quadratic terms in the energy dependence of the cross sections and solving a truncated system of moments equations.

\section{The Boltzmann equation and moments equations}

We adopt here a one-dimensional electron scattering model and define

$$
f(x, v) \mathrm{d} v=f\left[x,(2 \varepsilon / m)^{1 / 2}\right] \mathrm{d} v=F(x, \varepsilon) \mathrm{d} v
$$

where $\varepsilon=m v^{2} / 2$ as the density of those electrons in the discharge at point $x$, which are characterised by velocities in the interval $(v, v+\mathrm{d} v)$. The distribution $F(x, \varepsilon)$ obeys the Boltzmann equation, which in our case can be written in the following form:

$$
\begin{aligned}
v \frac{\partial F}{\partial x} \mathrm{~d} v+\frac{e E}{m} & \frac{\partial F}{\partial v} \mathrm{~d} v=F\left(x, \varepsilon^{\prime}\right) p Q_{\mathrm{r} 0}\left(\varepsilon^{\prime}\right) v^{\prime} \mathrm{d} v^{\prime}-F(x, \varepsilon) p Q_{\mathrm{r} 0}(\varepsilon) v \mathrm{~d} v \\
& +m v \mathrm{~d} v p \int_{0}^{\infty} F\left(x, \varepsilon^{\prime \prime}\right) Q_{\mathrm{i} 0}\left(\varepsilon^{\prime \prime}\right) \varphi\left(\varepsilon^{\prime \prime}, \varepsilon\right) v^{\prime \prime} \mathrm{d} v^{\prime \prime} \\
& +m v \mathrm{~d} v p \int_{0}^{\infty} F\left(x, \varepsilon^{\prime \prime}\right) Q_{\mathrm{i} 0}\left(\varepsilon^{\prime \prime}\right) \eta\left(\varepsilon^{\prime \prime}, \varepsilon\right) v^{\prime \prime} \mathrm{d} v^{\prime \prime}-F(x, \varepsilon) p Q_{\mathrm{i} 0}(\varepsilon) v \mathrm{~d} v
\end{aligned}
$$

where $p$ is the pressure of the gas, $\varepsilon^{\prime}=\varepsilon+\varepsilon_{1}$, and $Q_{\mathrm{r} 0}$ and $Q_{\mathrm{i} 0}$ are the total cross sections at normal conditions $\left(p=1 \mathrm{Torr}, T=273^{\circ} \mathrm{C}\right)$. In reducing the collision term (3) we combined all the electron-atom excitation processes (the total cross section $Q_{\mathrm{r} 0}(\varepsilon)$ ) to define an average excitation energy $\varepsilon_{1}$ and assumed that

$$
Q_{\mathrm{r} 0}(\varepsilon)=0 \quad \varepsilon<\varepsilon_{1} .
$$

Similarly $Q_{\mathrm{i} 0}$ in (3) is the total ionisation cross section, and if we define $\varepsilon_{2}$ as the average energy loss in ionisation we assume

$$
Q_{\text {i0 }}(\varepsilon)=0 \quad \varepsilon<\varepsilon_{2} .
$$

In equation (3), the function $\eta$ is defined so that $\eta\left(\varepsilon^{\prime \prime}, \varepsilon\right) \mathrm{d} \varepsilon$ is the probability of an event in which the energy of the secondary electron in the ionisation process belongs to the 
interval $(\varepsilon, \varepsilon+\mathrm{d} \varepsilon)$ if the primary electron has energy $\varepsilon^{\prime \prime}$. Similarly, $\varphi\left(\varepsilon^{\prime \prime}, \varepsilon\right) \mathrm{d} \varepsilon$ describes the probability of the energy of the primary electron after the ionisation belonging to the interval $(\varepsilon, \varepsilon+\mathrm{d} \varepsilon)$ if initially this energy was $\varepsilon^{\prime \prime}$. Obviously

$$
\eta\left(\varepsilon^{\prime \prime}, \varepsilon\right)=\varphi\left(\varepsilon^{\prime \prime}, \varepsilon\right)=0 \quad \varepsilon>\varepsilon^{\prime \prime}-\varepsilon_{\mathrm{i}}
$$

and

$$
\int_{0}^{\varepsilon^{\prime \prime}-\varepsilon_{\mathrm{i}}} \eta\left(\varepsilon^{\prime \prime}, \varepsilon\right) \mathrm{d} \varepsilon=\int_{0}^{\varepsilon^{\prime \prime}-\varepsilon_{\mathrm{i}}} \varphi\left(\varepsilon^{\prime \prime}, \varepsilon\right) \mathrm{d} \varepsilon=1
$$

where $\varepsilon_{\mathrm{i}}$ is the ionisation energy of the gas.

Moreover, by definition,

$$
\eta\left(\varepsilon^{\prime \prime}, \varepsilon\right)=\varphi\left(\varepsilon^{\prime \prime}, \varepsilon^{\prime \prime}-\varepsilon_{2}-\varepsilon\right) .
$$

Note that since $\eta\left(\varepsilon^{\prime \prime}, \varepsilon\right) \mathrm{d} \varepsilon=\eta\left(\varepsilon^{\prime \prime}, \varepsilon\right) m v \mathrm{~d} v$, the probability of the event in which the secondary electron has $v=0$ vanishes. Similar conditions also characterise the primary electron. Thus, ionisation events do not contribute the distribution function $f(x, v)$ to the interval $\mathrm{d} v$ around $v=0$. Taking (4) into account, we also conclude that excitation events also do not increase the number of electrons in this interval. Therefore, finally,

$$
f(x, 0)=0 \quad x \neq 0
$$

and, as usual,

$$
f(x, \infty)=0 .
$$

Direct numerical solution of (3) for $f$ is rather complex. On the other hand, in experiments, one usually studies various moments of the distribution function such as the particle current density $j(x)$ energy flux $\tilde{\varepsilon}(x)$ etc. These quantities are related to $f(x, v)$ via

$$
\begin{aligned}
& j(x)=\int_{0}^{x} v F(x, \varepsilon) \mathrm{d} v \\
& \bar{\varepsilon}(x)=\int_{0}^{\infty} \varepsilon v F(x, \varepsilon) \mathrm{d} v
\end{aligned}
$$

and obey rather simple moments equations. Indeed, by integrating (3) with respect to $v$, after some simple algebra, we get

$$
\mathrm{d} j / \mathrm{d} x=\bar{\nu}_{\mathrm{i}}(x)
$$

where

$$
\bar{\nu}_{\mathrm{i}}(x)=p \int_{0}^{\infty} F(x, \varepsilon) Q_{\mathrm{i} 0}(\varepsilon) v \mathrm{~d} v .
$$

Equation (11) defines the average flux of the energy $\varepsilon$ and not the average quantities themselves. Equation (13) defines the number of ionisations in the units of volume per second. Equation (12) is, in fact, the continuity equation for our problem and $\bar{\nu}_{\mathrm{i}}(x)$ gives the average ionisation frequency in unit volume. Next, on multiplying (3) by $\varepsilon$ and again intergrating the resulting equation with respect to $\nu$ and using the properties of $\varphi$ and $\eta$, we get the second-moment equation

$$
\mathrm{d} \bar{\varepsilon} / \mathrm{d} x=e E j-\varepsilon_{1} \bar{\nu}_{\mathrm{r}}(x)-\varepsilon_{2} \bar{\nu}_{\mathrm{i}}(x)
$$


where

$$
\bar{\nu}_{\mathrm{r}}=p \int_{0}^{\infty} F(x, \varepsilon) Q_{\mathrm{r} 0}(\varepsilon) v \mathrm{~d} v .
$$

Equation (14) describes the energy balance in the growing current and $\bar{\nu}_{\mathrm{r}}$ is the average number of excitations per second in unit volume. The solution of (12) and (14) requires the knowledge of $\bar{\nu}_{\mathrm{i}}(x)$ and $\bar{\nu}_{\mathrm{r}}(x)$. The next two sections describe two possible approaches in approximating these functions, thus allowing the solution of the moments equations.

\section{Linear energy dependence of inelastic cross sections}

Assume, at this point, that the ionisation and the total excitation cross sections depend linearly on the electron energy

$$
Q_{\mathrm{i} 0}(\varepsilon)=a \varepsilon \quad Q_{\mathrm{r} 0}(\varepsilon)=k \varepsilon
$$

We will discuss this assumption at the end of this section. Then

$$
\bar{\nu}_{\mathrm{i}}(x)=p a \bar{\varepsilon} \quad \bar{\nu}_{\mathrm{r}}(x)=p k \bar{\varepsilon}
$$

and equations (12) and (14) reduce to the following complete set of equations:

$$
\begin{aligned}
& \mathrm{d} j / \mathrm{d} x=p a \bar{\varepsilon} \\
& \mathrm{d} \bar{\varepsilon} / \mathrm{d} x=e E(x) j-p\left(\varepsilon_{1} k+\varepsilon_{2} a\right) \bar{\varepsilon}
\end{aligned}
$$

The electron flux $j$ thus satisfies the following second-order ordinary differential equation

$$
\mathrm{d}^{2} j / \mathrm{d} x^{2}+p\left(\varepsilon_{1} k+\varepsilon_{2} a\right) \mathrm{d} j / \mathrm{d} x-e p a E(x) j=0 .
$$

Assume now that $E(x)=E=$ constant. Then the solution of $(20)$ is

$$
j(x)=C_{1} \mathrm{e}^{\alpha_{1} x}+C_{2} \mathrm{e}^{\alpha_{2} x}
$$

where $\alpha_{1,2}$ are the roots of the quadratic equation

$$
\alpha^{2}+p\left(\varepsilon_{1} k+\varepsilon_{2} a\right)-e p a E=0
$$

or

$$
\frac{\alpha_{1,2}}{p}=-\frac{\varepsilon_{1} k+\varepsilon_{2} a}{2} \pm\left[\left(\frac{\varepsilon_{1} k+\varepsilon_{2} a}{2}\right)^{2}+a \frac{e E}{p}\right]^{1 / 2}
$$

Obviously, $\alpha_{1}>0, \alpha_{2}<0$ and $\left|\alpha_{2}\right|>\alpha_{1}$. The constants $C_{1}$ and $C_{2}$ in (21) are found from the initial conditions $j(0)=j_{0}$ and $\left.\bar{\varepsilon}\right|_{x=0}=(1 / p a) \mathrm{d} j / \mathrm{d} x=0$. This yields

$$
C_{1}=\alpha_{1} j_{0} /\left(\alpha_{1}-\alpha_{2}\right) \quad C_{2}=\alpha_{2} j_{0} /\left(\alpha_{2}-\alpha_{1}\right) .
$$

At large enough values of $p x(21)$ thus becomes

$$
j_{x}(x)=\left(j_{0} \alpha_{1} / \alpha_{1}-\alpha_{2}\right) \exp \left[\left(\alpha_{1} / p\right) p x\right]
$$

where $\alpha_{1}$ is the conventional first Townsend ionisation coefficient.

The asymptotic (large-px) expression for $\bar{\varepsilon}(x)$ can now be found from (18):

$$
\varepsilon_{x}(x)=\left[\left(j_{0} \alpha_{1} / p\right) / a\left(\alpha_{1}-\alpha_{2}\right)\right] \exp \left(\alpha_{1} x\right)
$$


and, therefore, the asymptotic value of the average energy per particle in the flux becomes

$$
\varepsilon_{0}=\varepsilon_{x} / j_{x}=\alpha_{1} / p a .
$$

Let us now discuss the validity of the linear approximation, for the inelastic cross sections. This approximation is always valid at relatively small electron energies. As an example we show $Q_{\mathrm{i} 0}(\varepsilon)$ and $Q_{\mathrm{r} 0}(\varepsilon)$ for $\mathrm{A}$ in figure 1 . In table 1 we also present the values

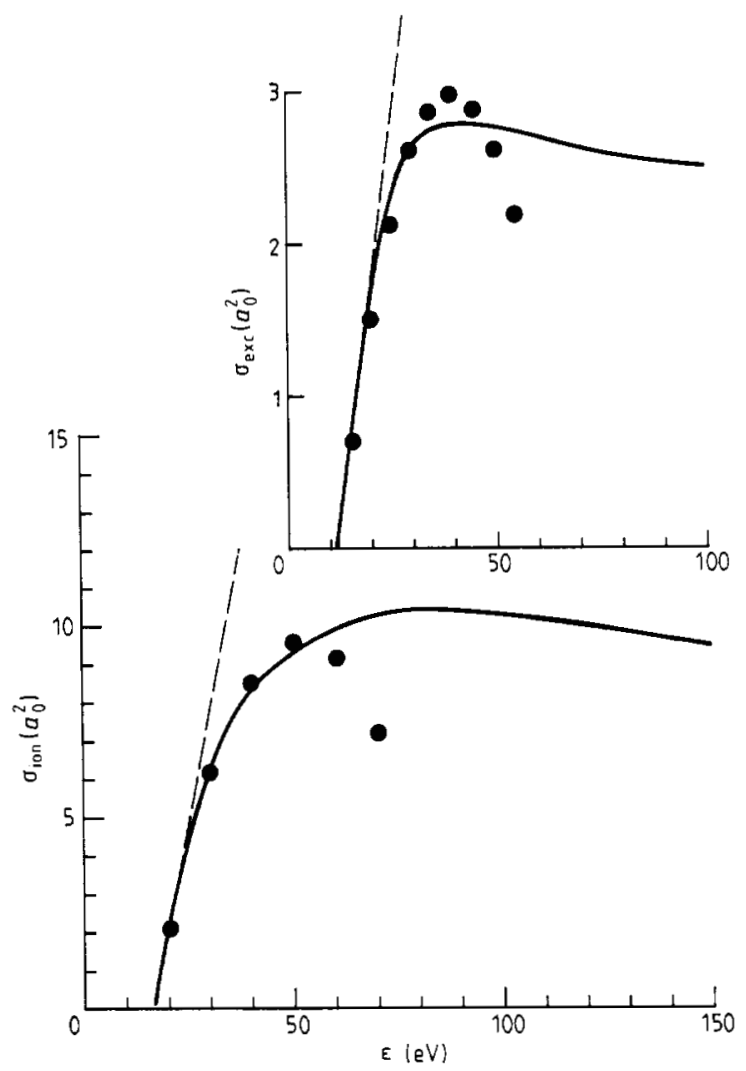

Figure 1. The cross section of ionisation and total cross section of inelastic collisions for A with $a_{0}=0.528 \times 10^{-8} \mathrm{~cm}$. Full curves: experimental results; broken lines: the linear approximation; full circles: the quadratic approximation.

Table 1. Constants characterising ionisation and inelastic efficiencies in various gases.

\begin{tabular}{lllllll}
\hline & $\begin{array}{l}k \\
\left((\mathrm{~cm} \mathrm{Torr} \mathrm{eV})^{-1}\right)\end{array}$ & $\begin{array}{l}k \\
\left((\mathrm{~cm} \text { Torr eV })^{-1}\right)\end{array}$ & $\begin{array}{l}c \\
\left((\mathrm{~cm} \mathrm{Torr})^{-1} \mathrm{eV}^{-2}\right)\end{array}$ & $\begin{array}{l}c_{1} \\
\left((\mathrm{~cm} \mathrm{Torr})^{-1} \mathrm{eV}^{-2}\right)\end{array}$ & $\begin{array}{l}\varepsilon_{1} \\
(\mathrm{eV})\end{array}$ & $\begin{array}{l}\varepsilon_{2} \\
(\mathrm{eV})\end{array}$ \\
\hline $\mathrm{Ne}$ & 0.047 & 0.027 & $1.8 \times 10^{-4}$ & $2.8 \times 10^{-4}$ & 16.5 & 21.6 \\
$\mathrm{~A}$ & 0.53 & 0.21 & $7.3 \times 10^{-3}$ & $3.7 \times 10^{-3}$ & 11.5 & 15.7 \\
$\mathrm{Kr}$ & 0.68 & 0.26 & $8.5 \times 10^{-3}$ & $4.5 \times 10^{-3}$ & 9.9 & 14.0 \\
$\mathrm{Xe}$ & 1.13 & 0.26 & 0.017 & $2.9 \times 10^{-3}$ & 8.3 & 12.1 \\
\hline
\end{tabular}




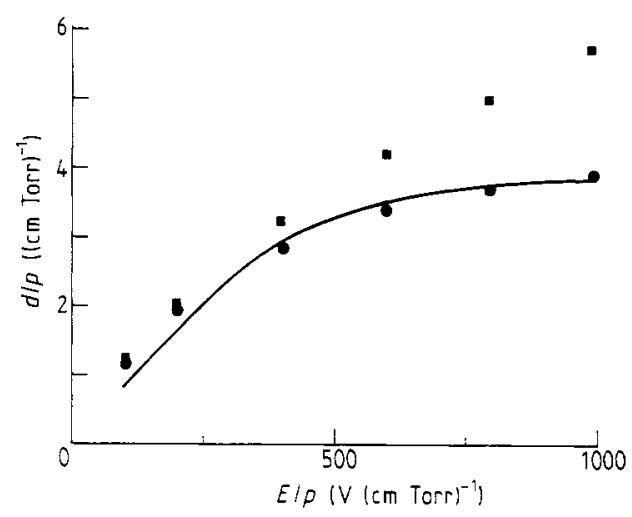

Figure 2. The first Townsend ionisation coefficient versus $E / p$ for Ne. Full curves: experimental results: full squares: the linear approximation; full circles: the quadratic approximation.

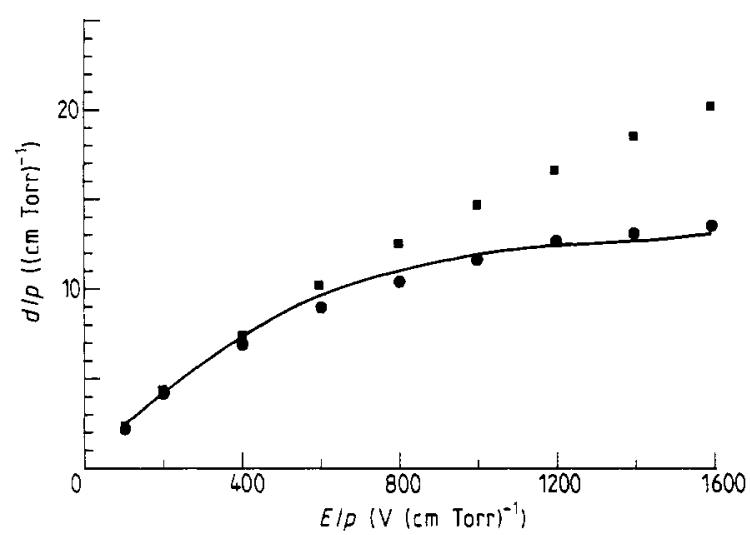

Figure 3. The first Townsend ionisation coefficient versus $E / p$ for $\mathrm{A}$. Full curves: experimental results; full squares: the linear approximation; full circles: the quadratic approximation.

of $a$ and $k$ (initial slopes of the ionisation and total excitation cross sections), for a variety of inert gases (der Heer et al 1979). In the same table we also show the values of $\varepsilon_{1}$ and $\varepsilon_{2}$. We used the excitation energy of the lowest level of an atom and the ionisation energy as an estimate for $\varepsilon_{1}$ and $\varepsilon_{2}$.

The first Townsend ionisation coefficient $\alpha_{1} / p$, found from equation (23) by using the data of table 1 for $\mathrm{Ne}, \mathrm{A}, \mathrm{Kr}$ and $\mathrm{Xe}$ is shown in figures $2-5$. One can see in these figures that, in general, the agreement between the theory and experiment at low values of $E / p$ is quite good. Nevertheless at high values of $E / p$ there is a systematic discrepancy between the computed and experimental curves. This discrepancy is due to the violation of the linear approximation of the cross sections at large average energies, characteristic to the regime of high values of $E / p$.

In the following section we will improve the theory by adding a quadratic correction to the energy dependence of the cross section. 


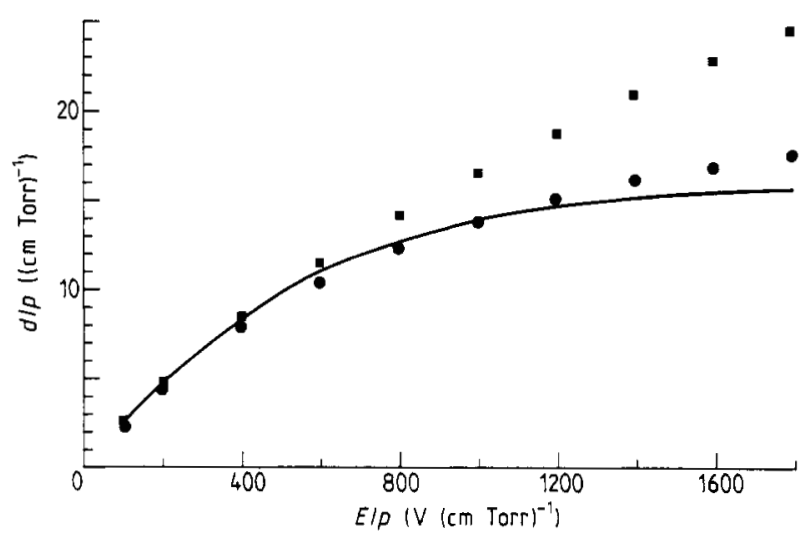

Figure 4. The first Townsend ionisation coefficient versus $E / p$ for $\mathrm{Kr}$. Full curves: experimental results; full squares: the linear approximation; full circles: the quadratic approximation.

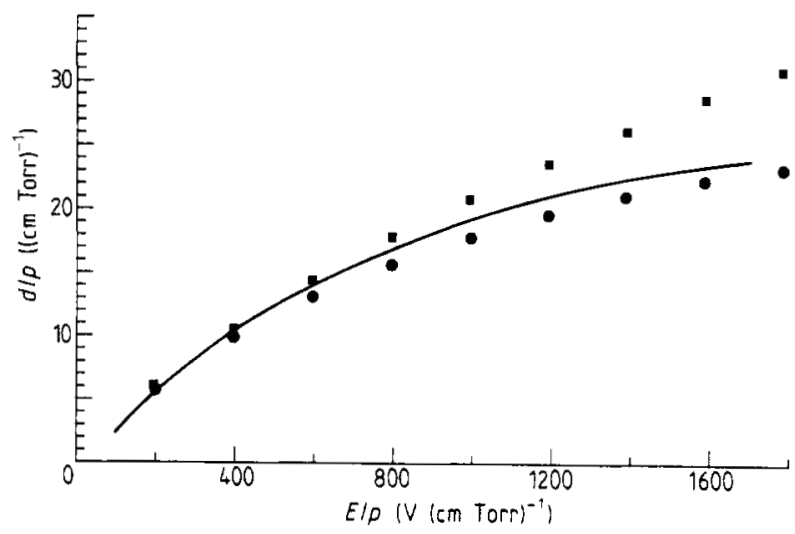

Figure 5. The first Townsend ionisation coefficient versus $E / p$ for Xe. Full curves: experimental results; full squares: the linear approximation; full circles: the quadratic approximation.

\section{Quadratic approximation of the inelastic cross sections}

Assume, now, that in contrast to (16)

$$
Q_{\mathrm{i} 0}=a \varepsilon-c \varepsilon^{2} \quad Q_{\mathrm{r} 0}=k \varepsilon-c_{1} \varepsilon^{2} .
$$

The values of $c$ and $c_{1}$ that are obtained from the experimental values (de Heer 1979) can be found in table 1 . Figure 1 for $A$ shows that in a certain energy range the use of (28) improves the agreement with the experimental cross sections. Similarly to (17) we now have:

$$
\bar{\nu}_{\mathrm{i}}=p a \bar{\varepsilon}-p c \overline{\varepsilon^{2}} \quad \bar{\nu}_{\mathrm{r}}=p k \bar{\varepsilon}-p c_{1} \overline{\varepsilon^{2}} \quad \varepsilon^{2}=\int \varepsilon^{2} F(x, \varepsilon) v \mathrm{~d} v
$$


By substituting these expressions into (18) and (19), we get

$$
\begin{aligned}
& \mathrm{d} j / \mathrm{d} x=p a \bar{\varepsilon}-p c \overline{\varepsilon^{2}} \\
& \mathrm{~d} \bar{\varepsilon} / \mathrm{d} x=e E j(x)-p\left(\varepsilon_{1} k+\varepsilon_{2} a\right) \bar{\varepsilon}+p\left(\varepsilon_{1} c_{1}+\varepsilon_{2} c_{2}\right) \overline{\varepsilon^{2}} .
\end{aligned}
$$

We can see that equations (30) and (31) are not sufficient for solving the problem in a quadratic approximation. To proceed, we have to derive an equation for $\overline{\varepsilon^{2}}$. The latter is found from the kinetic equation (3) by multiplying it by $\varepsilon^{2}$ and integrating with respect to $v$. The resulting equation involves integrals which can be evaluated only if the scattering function $\eta\left(\varepsilon^{\prime}, \varepsilon\right)$ is known. We will assume, in agreement with the experiment, that most of the secondary electrons in the ionisation process have energies much lower than that of the primary electron, and consequently we can approximate $\eta\left(\varepsilon^{\prime \prime}, \varepsilon\right)$ by the $\delta$-function $\delta(\varepsilon)$. This yields the following equation for $\overline{\varepsilon^{2}}$ :

$$
\begin{gathered}
\mathrm{d} \overline{\varepsilon^{2}} / \mathrm{d} x=\left(2 e E+p \varepsilon_{2}^{2} a+p \varepsilon_{1}^{2} k\right) \bar{\varepsilon}-p\left[2\left(\varepsilon_{1} k+\varepsilon_{2} a\right)+\varepsilon_{1}^{2} c_{1}+\varepsilon_{2}^{2} c\right] \overline{\varepsilon^{2}} \\
+2 p\left(\varepsilon_{1} c_{1}+\varepsilon_{2} c_{2}\right) \overline{\varepsilon^{3}} .
\end{gathered}
$$

This equation involves the higher moment $\overline{\varepsilon^{3}}$ so the system of moments equations is not closed. Nevertheless the following arguments allows us to perform the closure. The quadratic approximation for the cross sections is only valid if the quadratic terms are small compared with the linear terms (otherwise we should continue the expansion and include cubic terms etc). Therefore, naturally in using (29), we assumed that

$$
c \overline{\varepsilon^{2}} \ll a \bar{\varepsilon} \quad c_{1} \overline{\varepsilon^{2}} \ll k \bar{\varepsilon} .
$$

Thus, to the lowest significant order, equation (32) can be solved by taking $c=c_{1}=0$. In this approximation our system of moments equations becomes closed.

Defining a new variable $z=p x$ and letting

$$
\begin{aligned}
& \gamma=e E / p \quad A_{1}=\varepsilon_{1} k+\varepsilon_{2} a \quad A_{2}=\varepsilon_{1} c_{1}+\varepsilon_{2} c \\
& A_{3}=\varepsilon_{2}^{2} a+\varepsilon_{1}^{2} k \quad A_{+}=2 A_{1}
\end{aligned}
$$

equations $(30)-(32)$ become

$$
\begin{aligned}
& \mathrm{d} j / \mathrm{d} z=a \bar{\varepsilon}-c \overline{\varepsilon^{2}} \\
& \mathrm{~d} \bar{\varepsilon} / \mathrm{d} z=\gamma j-A_{1} \bar{\varepsilon}+A_{2} \overline{\varepsilon^{2}} \\
& \mathrm{~d} \overline{\varepsilon^{2}} / \mathrm{d} z=\left(2 \gamma+A_{3}\right) \bar{\varepsilon}-A_{4} \overline{\varepsilon^{2}}
\end{aligned}
$$

which must be solved subject to the following initial conditions:

$$
j(0)=j_{0} \quad \bar{\varepsilon}(0)=0 \quad \overline{\varepsilon^{2}}(0)=0 .
$$

We seek solutions of the form $B \mathrm{e}^{\beta z}$ of (35)-(37). This yields the characteristic equation for $\beta$

$$
\left(A_{4}+\beta\right)[\beta(\beta+A)-a \gamma]+\left(2 \gamma+A_{3}\right)\left(c \gamma-\beta A_{2}\right)=0 .
$$

The second term in (39) is of an order of magnitude proportional to $c$ (or $c_{1}$ ). In the zero approximation (the linear approximation of the cross sections) we therefore neglect this term. Then $\beta$ satisfies a quadratic equation

$$
\beta(\beta+A)-a \gamma=0
$$

which, of course, coincides with (22) and its largest root $\beta_{10}$, having the meaning of the 
first Townsend ionisation coefficient, in new notation becomes

$$
\beta_{10}=-A_{1} / 2+\left[\left(A_{1} / 2\right)^{2}+a \gamma\right]^{1 / 2} .
$$

In the first order, taking into account the terms of order $c$ in (39), we rewrite (29)

$$
\beta\left(\beta+A_{1}\right)-a \gamma+\left[\left(2 \gamma+A_{3}\right) /\left(A_{4}+\beta_{10}\right)\right]\left(c \gamma-\beta_{10} A_{2}\right)=0 .
$$

By using the notation $\Delta=\left(2 \gamma+A_{3}\right)\left(c \gamma-\beta_{10} A_{2}\right) /\left(A_{4}+\beta_{10}\right)$, the solution of (39), in the first order then becomes

$$
\beta_{1}=-A_{1} / 2+\left[\left(A_{1} / 2\right)^{2}+a \gamma-\Delta\right]^{1 / 2} .
$$

The numerical values of $\beta_{1} / p$ found by using equation (43) are compared in figures 2-5 with the experimental results for the first Townsend ionisation coefficient (Brown 1959, Fletcher and Davies 1963).

The comparison for $\mathrm{Ne}, \mathrm{A}, \mathrm{Kr}$ and $\mathrm{Xe}$ shows an excellent agreement with the experiment for all values of $E / p$. The validity of condition (33) used in the described perturbation scheme depends on the smallness of the ratio

$$
\eta=c \overline{\varepsilon^{2}} / a \bar{\varepsilon}
$$

Asymptotically the moments equations (35)-(37) yield

$$
\eta=(c / a)\left(2 \gamma+A_{3}\right) /\left(\beta_{10}+A_{4}\right)
$$

which, as can be checked, remains reasonably small in the entire range of the values of $E / p$.

\section{Discussion}

We now briefly discuss the peculiarities of the present calculation. The Townsend coefficient $\alpha$ in inert gases was calculated previously by a variety of other methods, such as the direct solution of the Boltzmann equation or by means of the Monte Carlo computer simulation (see, for example, Kucukarpaci et al (1981), Taniguchi et al (1978)). The present study presents a different approach to the problem which yields an analytic expression of $\alpha / p$ in a large range of the values of $E / p$ and is in good agreement with the experiment as can be seen in figures $2-5$.

The validity of the theory in such a large range of $E / p$ for a variety of gases needs an explanation. The reason for this is the fact that in spite of the fast acceleration of the electrons in the cases of extreme fields, the number of new slow electrons created in ionisations is sufficiently large. Therefore the bulk of the electrons in the avalanche is characterised by relatively small energies, usually below the energy of the maximum in the inelastic cross sections $\varepsilon_{\mathrm{m}}$. Thus the linear (or quadratic) approximation of the cross sections remains valid. On the other hand the validity range is limited from below by values of $E / p$ for which the one-dimensional scattering model becomes wrong and from above by such values of $E / p$ for which an electron is accelerated to energies larger than $\varepsilon_{\mathrm{m}}$ and creates on average, during its acceleration time, less than one new electron. For these values of $E / p$, the runaway effects become important and our approximation for the cross section becomes invalid. Within these limits the theory described should be applicable also in cases of molecular gases. Consideration of these cases, however, was beyond the scope of the present work. 


\section{References}

Brown S C 1959 Basic Data of Plasma Physics (New York: Wiley)

Fletcher J and Davies D E 1963 Proc. 6th Int. Conf. Phenomena in Ionised Gases, Paris 1963 (Paris: Association) p 217

Friedland L 1974 J. Phys. D: Appl. Phys. 72246

Friedland L and Kagan Yu M 1982 J. Phys. D: Appl. Phys. 151721

- 1983 J. Appl. Phys. 544947

de Heer F J, Jansen R H J and van der Kaay W 1979 J. Phys. B: At. Mol. Phys. 12279

Kucukarpaci H N. Saele H T and Lucas J J 1981 J. Phys. D: Appl. Phys. 149

Taniguchi T. Tagashira H and Sakai Y 1978 J. Phys. D: Appl. Phys. 111757 